\title{
Epitope Mapping of the Anti-Diacylglycerol Kinase Monoclonal Antibody DhMab-4 for Immunohistochemical Analysis
}

\author{
Teizo Asano,, Masato Sano, ${ }^{1}$ Junko Takei, ${ }^{1}$ Yusuke Sayama, ${ }^{1}$ Mika K. Kaneko, and Yukinari Kato ${ }^{1,2}$
}

\begin{abstract}
Diacylglycerol kinase (DGK) plays a pivotal role in intracellular signaling pathways in mammals. Activated G protein-coupled receptor activates phospholipase C (PLC) through heterotrimeric G protein, following which PLC hydrolyzes phosphatidylinositol 4,5-bisphosphate $\left(\mathrm{PIP}_{2}\right)$ into diacylglycerol (DG) and inositol 1,4,5trisphosphate $\left(\mathrm{IP}_{3}\right)$. DGK catalyzes DG phosphorylation to produce phosphatidic acid. DG and phosphatidic acid function as second messengers and their intracellular concentrations are regulated by DGK; therefore, DGK plays an important role in regulating many biological processes. There are ten DGK isozymes, of which DGK $\eta$ is classified as a type II DGK. Reports have shown that DGK $\eta$ is associated with several diseases; for example, it is highly expressed in the hippocampus and cerebellum and is a key element in bipolar disorder. Although a DGK $\eta$-specific monoclonal antibody $(\mathrm{mAb})$ is necessary to reveal the association between the expression of DGK $\eta$ and diseases, an anti-DGK $\eta \mathrm{mAb}$ for immunohistochemistry has not yet been established.

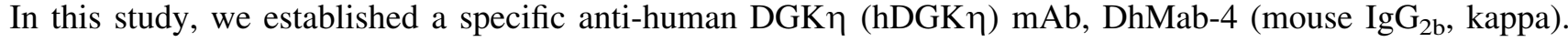
DhMab-4 strongly stained Purkinje cells of human cerebellum in immunohistochemistry analysis. For epitope mapping of DhMab-4, we produced deletion or point mutants of hDGK $\eta$ and performed western blotting to determine the binding epitope of DhMab-4. DhMab-4 reacted with dN745 mutant but not with dN750 mutant, indicating that the N-terminus of the DhMab-4 epitope is located between amino acids 745 and 750 . More detailed analysis using point mutants demonstrated that five mutants, that is, D747A, P748A, F749A, G750A, and T752A, were not detected by DhMab-4. These results indicate that Asp747, Pro748, Phe749, Gly750, and Thr752 are important for DhMab-4 binding to hDGK $\eta$.
\end{abstract}

Keywords: DGKh, DhMab-4, monoclonal antibody

\section{Introduction}

D IACYLGLYCEROL KINASE (DGK) plays a key role in the $\mathrm{G}$ protein-mediated signaling pathway, which is an important signaling cascade in mammalian cells. ${ }^{(1,2)}$ Activated $\mathrm{G}$ protein-coupled receptor catalyzes a guanine nucleotide exchange on the alpha subunit of the heterotrimeric $\mathrm{G}$ protein and resulting in its activation. An activated $\mathrm{G}$ protein subsequently activates phospholipase C (PLC). ${ }^{(3,4)}$ PLC hydrolyzes phosphatidylinositol 4,5-bisphosphate $\left(\mathrm{PIP}_{2}\right)$ into diacylglycerol (DG) and inositol 1,4,5-trisphosphate $\left(\mathrm{IP}_{3}\right)$, which functions as a second messenger. $\mathrm{IP}_{3}$ diffuses into the cytosol and increases $\mathrm{Ca}^{2+}$ concentration in the cytoplasm, whereas DG remains on the plasma membrane and activates protein kinase $\mathrm{C}(\mathrm{PKC}) .{ }^{(5-8)} \mathrm{DGK}$ is an enzyme that phosphorylates DG and produces phosphatidic acid. ${ }^{(1,2)}$ Phosphatidic acid also functions as a second messenger, which mediates intracellular $\mathrm{Ca}^{2+}$ levels and regulates the mTORmediated signaling pathway. ${ }^{(9,10)}$ Therefore, DGK regulates two signaling pathways to control a variety of biological functions. ${ }^{(11)}$

The DGK family consists of 10 isozymes in mammalian species and each isozyme contains 2 to $3 \mathrm{C} 1$ domains, while sharing a common catalytic domain. ${ }^{(1)}$ The DGK family is further divided into five subtypes according to their subtypespecific functional domains. ${ }^{(12)}$ DGK $\eta$ was first cloned from hamster and is classified as a type II DGK. It exhibits pleckstrin homology $(\mathrm{PH})$ domain and contains two $\mathrm{C} 1$ domains, a catalytic domain and an accessory domain. ${ }^{(13)}$ It is known that the PH domain is a phosphatidylinositol binding domain. The $\mathrm{PH}$ domain of DGK $\eta$ binds to $\mathrm{PIP}_{2}$ strongly and highly selectively, thereby regulating the subcellular localization of DGK $\eta .{ }^{(14)}$ There are two splicing variants of DGK $\eta$, DGK $\eta 1$, and DGK $\eta 2$. DGK $\eta 2$ has a sterile alpha

\footnotetext{
${ }^{1}$ Department of Antibody Drug Development, Tohoku University Graduate School of Medicine, Sendai, Japan.

${ }^{2}$ New Industry Creation Hatchery Center, Tohoku University, Sendai, Japan.
} 
motif domain at its carboxyl terminus, while the DGK $\eta 1$ variant is devoid of this domain. ${ }^{(15)}$

In this study, we immunized mice with a recombinant

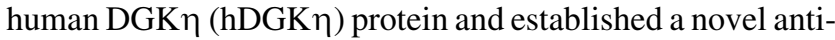
hDGK $\eta$ monoclonal antibody (mAb), DhMab-4, which may be used for immunohistochemistry. We also performed epitope mapping to reveal the binding epitope of DhMab-4 using deletion and point mutations of hDGK $\eta$.

\section{Materials and Methods}

\section{Plasmid preparation}

Synthesized DNA (Eurofins Genomics KK, Tokyo, Japan) encoding hDGK $\eta$ (Accession No.: AB078967) plus a C-terminal PA tag (GVAMPGAEDDVV), ${ }^{(16,17)}$ which is recognized by an anti-PA tag mAb (NZ-1), ${ }^{(18)}$ was subcloned into the expression vector, pMAL-c2 (New England Biolabs, Inc., Beverly, MA), using the In-Fusion HD Cloning Kit (Takara Bio, Inc., Shiga, Japan). The resultant construct was named pMAL-c2-hDGK $\eta$-PA. The deletion mutants of hDGK $\eta$ were produced by PCR and subcloned into pMAL-c2 with a PA tag using the In-Fusion HD Cloning Kit. The substitution of hDGK $\eta$ amino acids with alanine at dN705 of hDGK $\eta$ was performed using the QuikChange Lightning Site-Directed Mutagenesis Kit (Agilent Technologies, Inc., Santa Clara, CA). These constructs were verified by direct DNA sequencing.

\section{Production of recombinant DGK $\eta$ protein}

Competent Escherichia coli TOP-10 cells (Thermo Fisher Scientific, Inc., Waltham, MA) were transformed with the plasmid pMAL-c2-hDGK $\eta-P A$. They were then cultured overnight at $37^{\circ} \mathrm{C}$ in the LB medium (Thermo Fisher Scientific, Inc.) containing $100 \mu \mathrm{g} / \mathrm{mL}$ ampicillin (SigmaAldrich Corp., St. Louis, MO). Cell pellets were resuspended in phosphate-buffered saline (PBS) with $1 \%$ Triton X-100 and $50 \mu \mathrm{g} / \mathrm{mL}$ aprotinin (Sigma-Aldrich Corp.). After sonication, the crude extracts were collected by centrifugation $\left(9000 \mathrm{~g}, 30\right.$ minutes, $\left.4^{\circ} \mathrm{C}\right)$. The lysates were passed through a $0.45-\mu \mathrm{m}$ filter to remove any trace amounts of insoluble materials. Cleared lysates were mixed with NZ-1-Sepharose ( $1 \mathrm{~mL}$ bed volume) and incubated at $4{ }^{\circ} \mathrm{C}$ for 2 hours under gentle agitation. The resin was then transferred to a column and washed with $20 \mathrm{~mL}$ Tris-buffered saline (TBS) (pH 7.5). The bound protein was eluted with the PA tag peptide at room temperature in a stepwise manner $(1 \mathrm{~mL} \times 10)$.

\section{Hybridoma production}

DhMab-4 was produced using the mouse medial iliac lymph node method. The Animal Care and Use Committee of Tohoku University approved all of the animal experiments. Briefly, B6D2F1/Slc mice (Japan SLC, Inc., Shizuoka, Japan) were immunized by injecting $33 \mu \mathrm{g}$ of pMAL-c2-hDGK $\eta$-PA protein, along with Freund's complete adjuvant (SigmaAldrich Corp.), into the footpad. Additional immunization with $60 \mu \mathrm{g}$ of pMAL-c2-hDGK $\eta$-PA protein was performed using the tailbase. The lymphocytes were fused with mouse myeloma Sp2/0-Ag14 cells using polyethylene glycol (PEG). The culture supernatants were screened using enzyme-linked immunosorbent assay for binding to pMAL-c2-hDGK $\eta$-PA protein.

\section{Immunohistochemical analyses}

Normal human cerebellum tissue was purchased from ProteoGenex, Inc. (Inglewood, CA). Histological sections of $4 \mu \mathrm{m}$ thickness were directly autoclaved in citrate buffer (pH 6.0; Nichirei Biosciences, Inc., Tokyo, Japan) for 20 minutes. After blocking with SuperBlock T20 (PBS) Blocking Buffer (Thermo Fisher Scientific, Inc.), the sections were incubated with DhMab-4 $(5 \mu \mathrm{g} / \mathrm{mL})$ for 1 hour at room temperature and treated using the EnVision+ Kit (Agilent Technologies, Inc.) for 30 minutes. Color was developed using 3,3'-diaminobenzidine tetrahydrochloride (DAB; Agilent Technologies, Inc.) for 2 minutes, and counterstaining was performed with hematoxylin (FUJIFILM Wako Pure Chemical Corporation, Osaka, Japan).

\section{Western blot analyses}

Lysates were boiled in sodium dodecyl sulfate sample buffer (Nacalai Tesque, Inc., Kyoto, Japan). The samples were electrophoresed on 5\%-20\% polyacrylamide gels (Nacalai Tesque, Inc.) and transferred onto a polyvinylidene
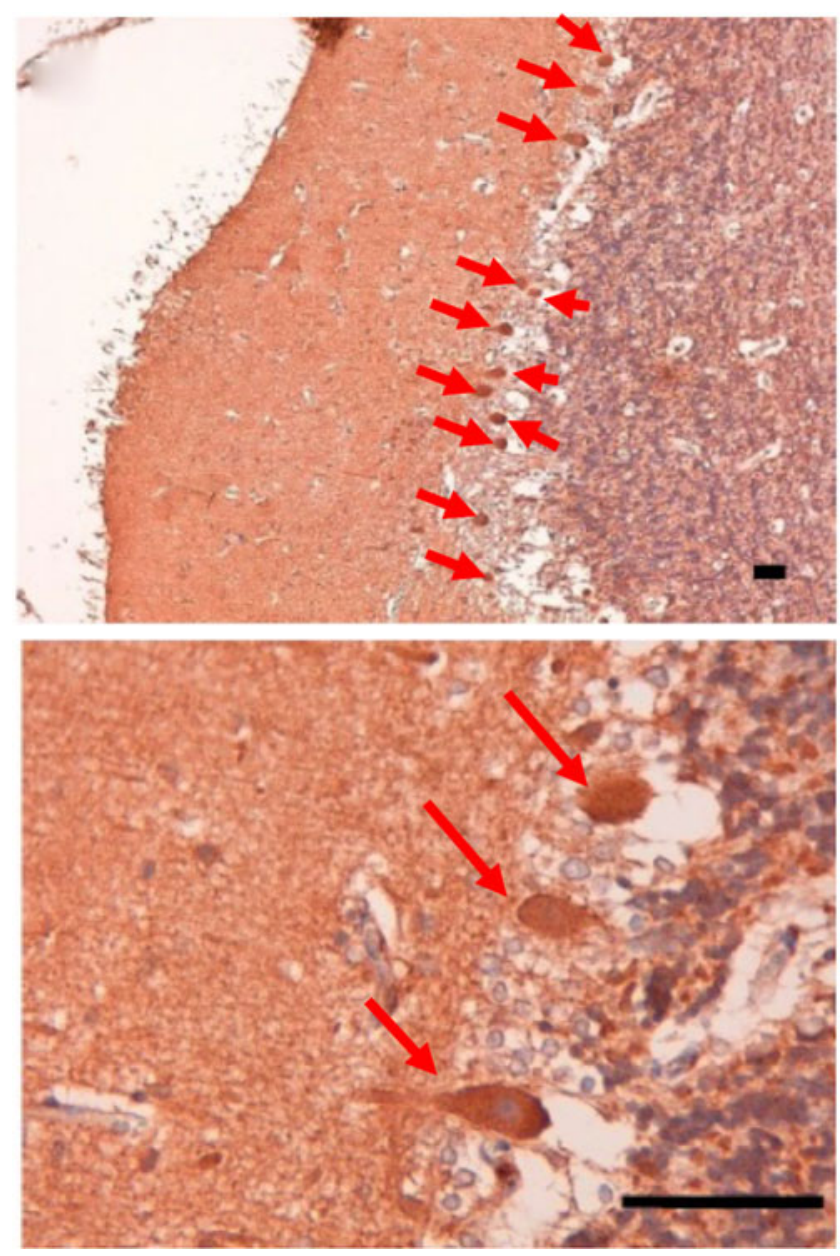

FIG. 1. Immunohistochemical analysis using DhMab-4 against human cerebellum. Tissue sections were incubated with DhMab-4 $(5 \mu \mathrm{g} / \mathrm{mL})$ for 1 hour at room temperature and treated using the EnVision+ kit for 30 minutes. Red arrows indicate Purkinje cells. Scale bar $=100 \mu \mathrm{m}$. 
difluoride (PVDF) membrane (Merck KGaA, Darmstadt, Germany). After blocking with $4 \%$ skim milk (Nacalai Tesque, Inc.) for 1 hour, the membrane was incubated with DhMab-4 or NZ-1 for 1 hour, followed by HRP-conjugated anti-mouse immunoglobulins (1:2000 dilution; Agilent Technologies, Inc.) or HRP-conjugated anti-rat IgG (1:10,000 dilution; Sigma-Aldrich Corp.) for 1 hour. The membrane was developed with the ImmunoStar LD Chemiluminescence Reagent (FUJIFILM Wako Pure Chemical Corporation) using the Sayaca-Imager (DRC Co., Ltd., Tokyo, Japan). All western blot procedures were performed at room temperature.

\section{Results}

In this study, we established DhMab-4 (mouse IgG $_{2 b}$, kappa), which can recognize hDGK $\eta$, but not other related isozymes such as DGK $\alpha, \mathrm{DGK} \gamma$, and DGK $\zeta$ in an enzymelinked immunosorbent assay (data not shown). DhMab-4 stained human cerebral Purkinje cells as determined by immunohistochemistry (Fig. 1).

We next performed epitope mapping to characterize the binding epitope of DhMab-4 using western blot analysis.
First, we produced a C-terminal deletion mutant (dC583) and an N-terminal deletion mutant (dN584) and investigated whether DhMab-4 recognizes either dC583 or dN584 by western blot analysis. The results indicated that DhMab-4 interacts with dN584 (Fig. 2A, left panel). We then produced additional $11 \mathrm{~N}$-terminal deletion mutants (dN600, dN650, dN700, dN750, dN800, dN850, dN900, dN950, dN1000,

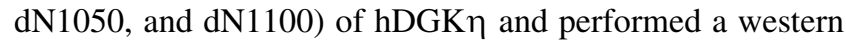
blot. As shown in Figure 2A (left panel), DhMab-4 recognized dN600, dN650, and dN700 but not dN750, dN800, dN850, dN900, dN950, dN1000, and dN1050. All of the deletion mutants were detected by an anti-PA tag mAb, NZ-1 (Fig. 2A, right panel). This result indicates that the N-terminus of the DhMab-4 epitope exists between 700 and 750 amino acids (aa) (Fig. 2B).

We next produced an additional nine $\mathrm{N}$-terminal deletion mutants of hDGK $\eta$ (dN705, dN710, dN715, dN720, dN725, dN730, dN735, dN740, and dN745). The western blot results showed that DhMab-4 and NZ-1 detected all of the deletion mutants (Fig. 3A), indicating that the N-terminus of the DhMab-4 binding epitope resides between amino acids 745 and 750 (Fig. 3B).
A

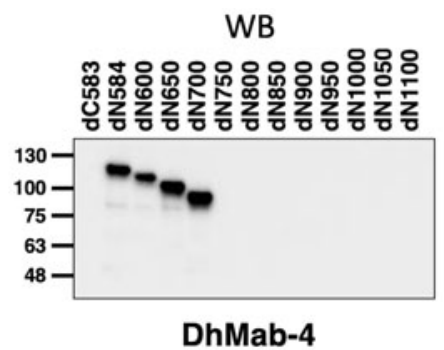

WB

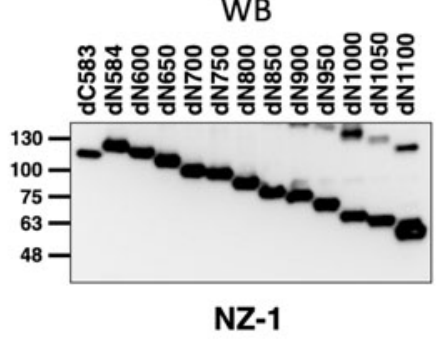

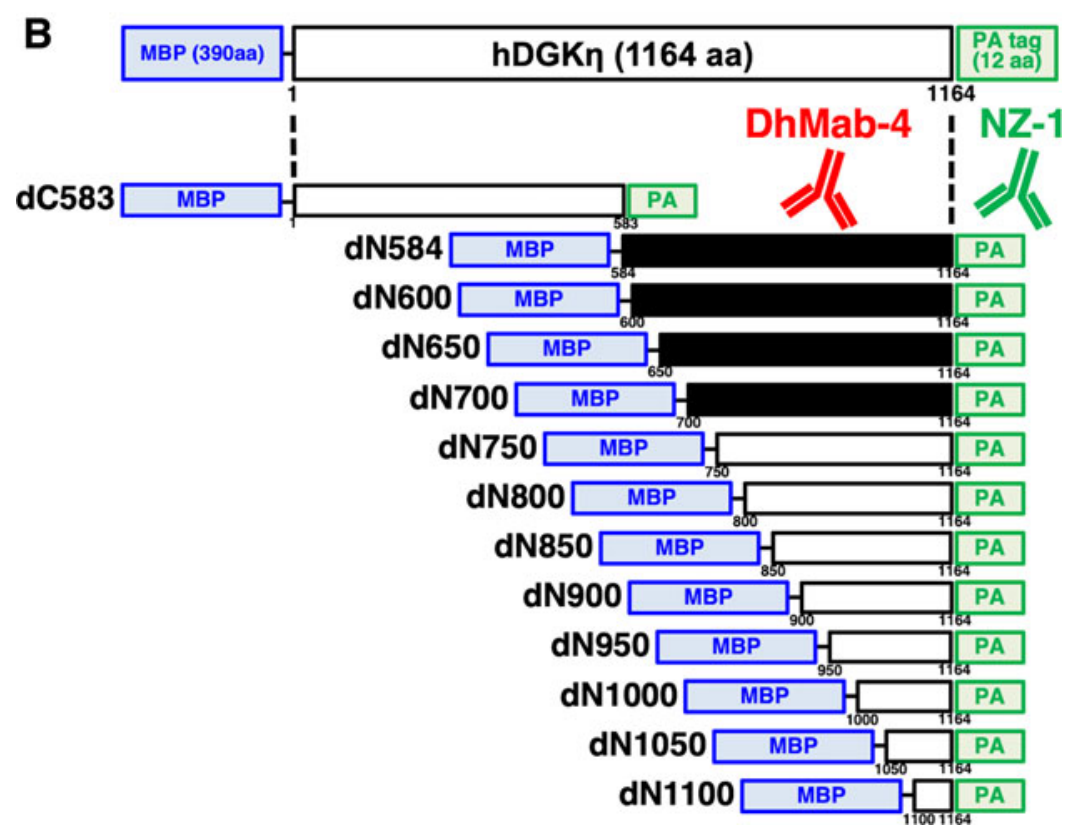

FIG. 2. Epitope mapping of DhMab-4 using deletion mutants of hDGKn. (A) Cell lysates of C- or N-terminal deletion mutants of $\mathrm{hDGK} \eta$ were electrophoresed and transferred onto a PVDF membrane. After blocking, the membrane was incubated with $1 \mu \mathrm{g} / \mathrm{mL}$ of DhMab-4 or anti-PA tag antibody (NZ-1). (B) Schematic illustration of epitope mapping of DhMab-4. Black bars, deletion mutants detected by DhMab-4. DGK, diacylglycerol kinase; hDGK $\eta$, human DGK $\eta$; MBP, maltose-binding protein; PVDF, polyvinylidene difluoride. 
A

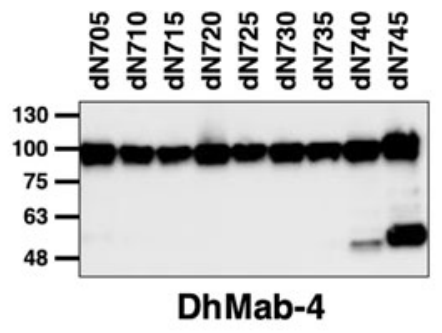

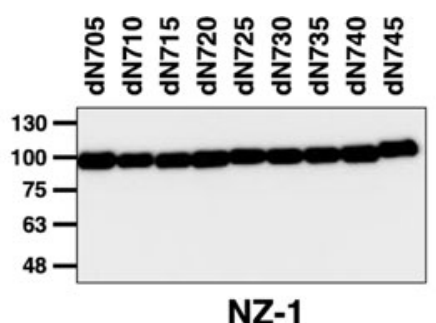

NZ-1

B

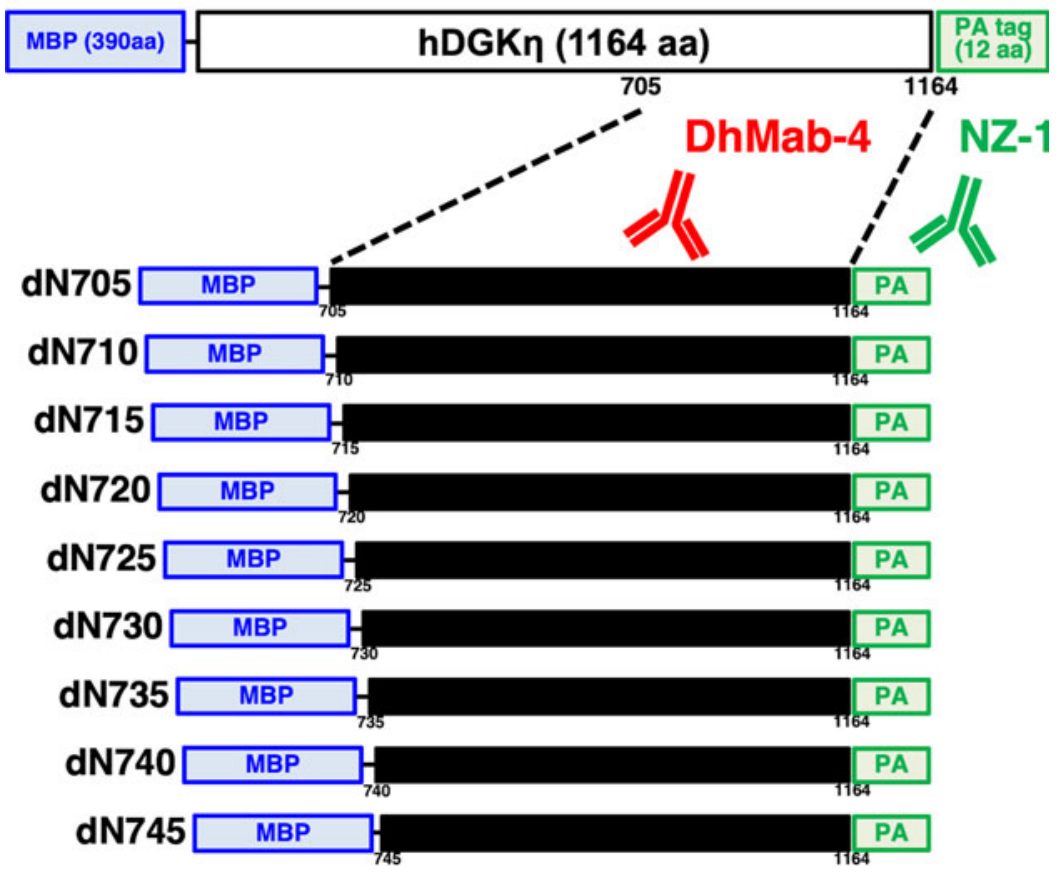

FIG. 3. Epitope mapping of DhMab-4 using N-terminal deletion mutants of hDGK $\eta$. (A) Cell lysates of N-terminal deletion mutants of $\mathrm{hDGK} \eta$ were electrophoresed and transferred onto a PVDF membrane. After blocking, the membrane was incubated with $1 \mu \mathrm{g} / \mathrm{mL}$ of DhMab-4 or anti-PA tag antibody (NZ-1). (B) Schematic illustration of epitope mapping of DhMab-4.

To further investigate the crucial epitope of DhMab-4, we produced constructs representing 15 point mutations within hDGK $\eta$ (N745A, I746A, D747A, P748A, F749A, G750A, A751G, T752A, P753A, F754A, I755A, D756A, P757A, D758A, and L759A). All hDGK $\eta$ point mutants were recognized by NZ-1 (Fig. 4A, right panel). In contrast, DhMab-4 did not recognize D747A, P748A, F749A, G750A, or T752A (Fig. 4A, left panel), indicating that DhMab-4 binds to DGK $\eta$ through these five amino acids. These results are summarized in Figure 4B.

\section{Discussion}

DGK plays an important role in signaling pathways by regulating the intracellular level of DG and phosphatidic acid, and it is considered a potential therapeutic target for some diseases. Recently, we established anti-DGK $\alpha,{ }^{(19,20)}$ anti-DGK $\gamma,{ }^{(21)}$ and anti-DGK $\zeta^{(22)} \mathrm{mAbs}$ and determined their respective binding epitopes. ${ }^{(20,23-25)}$ These mAbs are very useful for immunohistochemical analyses. However, we have not established anti-DGK $\eta$ mAbs for immunohistochemistry.
In this study, we established a novel anti-hDGK $\eta \mathrm{mAb}$, DhMab-4. DhMab-4 can detect hDGK $\eta$ in formalin-fixed paraffin-embedded human cerebellum tissue, indicating that DhMab-4 sensitively interacts with hDGK $\eta$ by immunohistochemical analysis (Fig. 1). We further demonstrated the binding epitope of DhMab-4 by western blot analyses. Five amino acids in hDGK $\eta$, Asp747, Pro748, Phe749, Gly750, and Thr752, are important for DhMab-4 binding to the hDGK $\eta$ protein. This epitope is located near the accessory domain of hDGKn (Fig. 4C). The amino acid sequence of this region is highly conserved among vertebrate DGK $\eta$; hence, DhMab-4 may be useful for detecting DGK $\eta$ in other species such as dog, chicken, lizard, and zebrafish.

DGK $\eta$ is highly expressed in the brain, especially in the hippocampus and cerebellum. ${ }^{(26)}$ The expression of DGK $\eta$ mRNA is increased in patients with bipolar disorder. ${ }^{(27,28)}$ Furthermore, behavioral tests using mice show that the behavioral profile is similar to human mania in DGK $\eta$ knockout mice. ${ }^{(29)}$ These results indicate that DGK $\eta$ plays a key role in bipolar disorder. In addition, DGK $\eta$ is also highly expressed in lung cancer containing EGFR mutations, and the knockdown of DGK $\eta$ has resulted in impaired growth of EGFR 

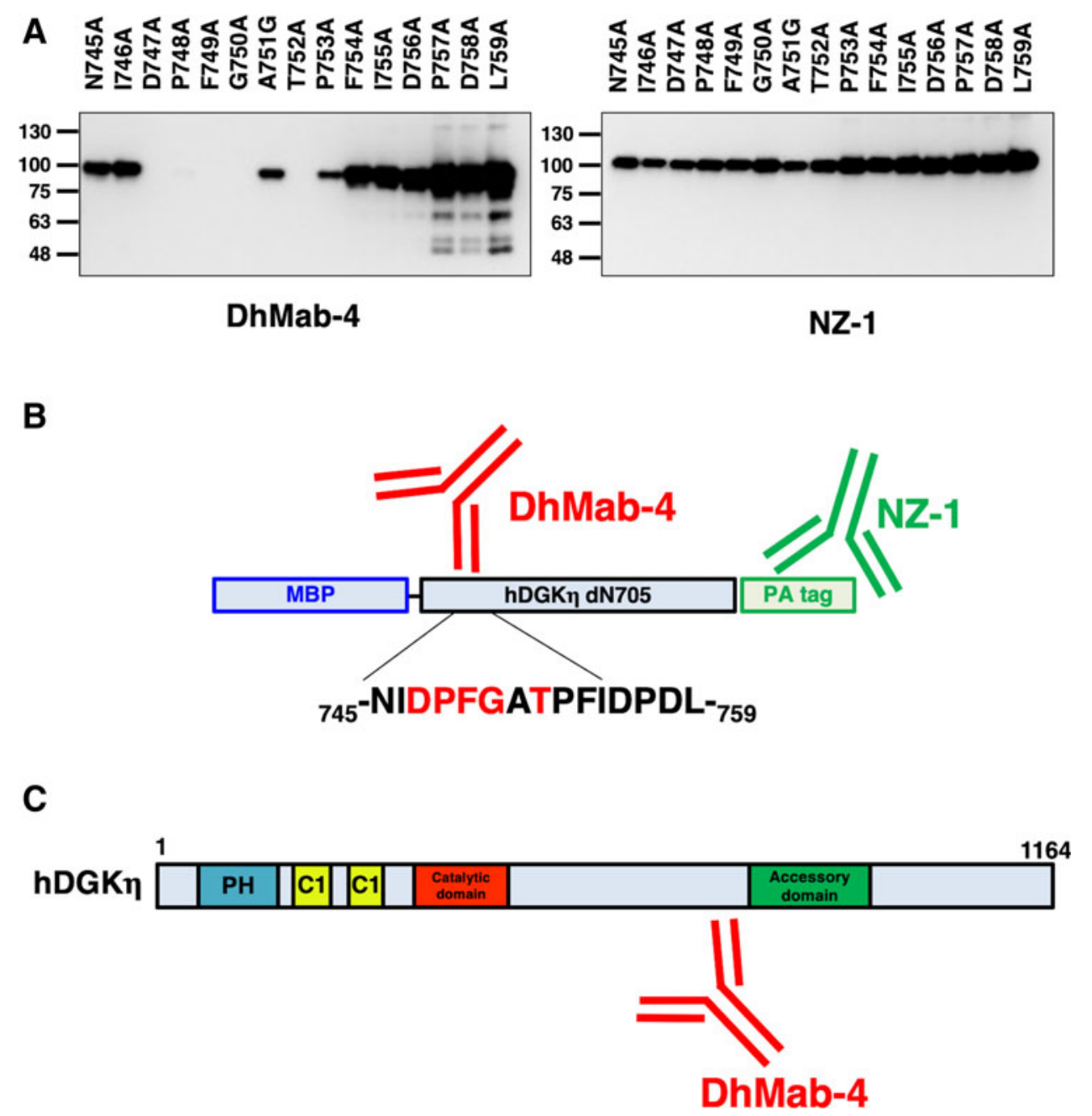

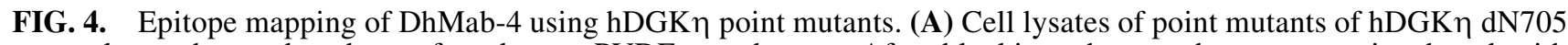
were electrophoresed and transferred onto PVDF membranes. After blocking, the membranes were incubated with $1 \mu \mathrm{g} / \mathrm{mL}$ of DhMab-4 or anti-PA tag antibody (NZ-1). (B) Schematic illustration of epitope mapping of DhMab-4 and anti-PA tag antibody (NZ-1). Asp747, Pro748, Phe749, Gly750, and Thr752 are important for DhMab-4 binding to

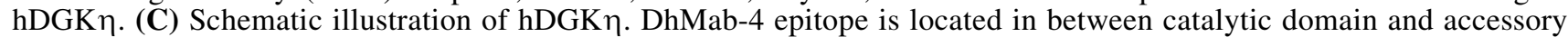
domain.

mutant cell lines. ${ }^{(30)}$ To understand the relationship between the expression of DGK $\eta$ and these diseases by immunohistochemical methods, a DGKn-specific mAb has been required. DhMab-4 can be used to analyze the expression and pathophysiological function of DGK $\eta$ and it represents a useful tool to study the molecular basis for various diseases.

\section{Author Disclosure Statement}

Y.K. received research funding from Ono Pharmaceutical Co., Ltd. The other authors have no conflict of interest.

\section{Funding Information}

This research was supported, in part, by AMED under Grant Numbers: JP19am0401013 (Y.K.), JP19am0101078 (Y.K.), and JP19ae0101028 (Y.K.) and by JSPS KAKENHI Grant Number 17K07299 (M.K.K.) and Grant Number 19K07705 (Y.K.).

\section{References}

1. Goto K, Hozumi Y, Nakano T, Saino SS, and Kondo H: Cell biology and pathophysiology of the diacylglycerol kinase family: Morphological aspects in tissues and organs. Int Rev Cytol 2007;264:25-63.

2. Topham MK, and Epand RM: Mammalian diacylglycerol kinases: Molecular interactions and biological functions of selected isoforms. Biochimica Biophys Acta Gen Subj 2009;1790:416-424.

3. Nakamura F, Kato M, Kameyama K, Nukada T, Haga T, Kato H, Takenawa T, and Kikkawa U: Characterization of Gq family G proteins GL1 alpha (G14 alpha), GL2 alpha (G11 alpha), and Gq alpha expressed in the baculovirusinsect cell system. J Biol Chem 1995;270:6246-6253.

4. Biddlecome GH, Berstein G, and Ross EM: Regulation of phospholipase C-beta1 by $\mathrm{Gq}$ and $\mathrm{m} 1$ muscarinic cholinergic receptor. Steady-state balance of receptor-mediated activation and GTPase-activating protein-promoted deactivation. J Biol Chem 1996;271:7999-8007. 
5. Berridge MJ, and Irvine RF: Inositol trisphosphate, a novel second messenger in cellular signal transduction. Nature 1984;312:315-321.

6. Newton AC: Regulation of protein kinase C. Curr Opin Cell Biol 1997;9:161-167.

7. Davey B, Parekh, Wolfgang Z, and Parker PJ: Multiple pathways control protein kinase $\mathrm{C}$ phosphorylation. EMBO J 2000;19:496-503.

8. Cosentino-Gomes D, Rocco-Machado N, and MeyerFernandes JR: Cell signaling through protein kinase $\mathrm{C}$ oxidation and activation. Int J Mol Sci 2012;13:10697-10721.

9. Fang Y, Vilella-Bach M, Bachmann R, Flanigan A, and Chen J: Phosphatidic acid-mediated mitogenic activation of mTOR signaling. Science 2001;294:1942-1945.

10. English D, Cui Y, and Siddiqui RA: Messenger functions of phosphatidic acid. Chem Phys Lipids 1996;80:117-132.

11. Merida I, Avila-Flores A, and Merino E: Diacylglycerol kinases: At the hub of cell signalling. Biochem J 2008;409:1-18.

12. Sakane F, Imai S, Kai M, Yasuda S, and Kanoh H: Diacylglycerol kinases: Why so many of them? Biochim Biophys Acta 2007;1771:793-806.

13. Klauck TM, Xu X, Mousseau B, and Jaken S: Cloning and characterization of a glucocorticoid-induced diacylglycerol kinase. J Biol Chem 1996;271:19781-19788.

14. Kume A, Kawase K, Komenoi S, Usuki T, Takeshita E, Sakai H, and Sakane F: The pleckstrin homology domain of diacylglycerol kinase eta strongly and selectively binds to phosphatidylinositol 4,5-bisphosphate. J Biol Chem 2016; 291:8150-8161.

15. Murakami T, Sakane F, Imai S, Houkin K, and Kanoh H: Identification and characterization of two splice variants of human diacylglycerol kinase eta. J Biol Chem 2003;278: 34364-34372.

16. Fujii Y, Kaneko M, Neyazaki M, Nogi T, Kato Y, and Takagi J: PA tag: A versatile protein tagging system using a super high affinity antibody against a dodecapeptide derived from human podoplanin. Protein Expr Purif 2014;95: 240-247.

17. Fujii Y, Matsunaga Y, Arimori T, Kitago Y, Ogasawara S, Kaneko MK, Kato Y, and Takagi J: Tailored placement of a turn-forming PA tag into the structured domain of a protein to probe its conformational state. J Cell Sci 2016;129:1512-1522.

18. Kato Y, Kaneko MK, Kuno A, Uchiyama N, Amano K, Chiba Y, Hasegawa Y, Hirabayashi J, Narimatsu H, Mishima $\mathrm{K}$, and Osawa M: Inhibition of tumor cell-induced platelet aggregation using a novel anti-podoplanin antibody reacting with its platelet-aggregation-stimulating domain. Biochem Biophys Res Commun 2006;349:1301-1307.

19. Nakano T, Ogasawara S, Tanaka T, Hozumi Y, Mizuno S, Satoh E, Sakane F, Okada N, Taketomi A, Honma R, Nakamura T, Saidoh N, Yanaka M, Itai S, Handa S, Chang YW, Yamada S, Kaneko MK, Kato Y, and Goto K: DaMab-2: Anti-human DGKalpha monoclonal antibody for immunocytochemistry. Monoclon Antib Immunodiagn Immunother 2017;36:181-184.

20. Sano M, Kaneko MK, Suzuki H, and Kato Y: Establishment and epitope mapping of anti-diacylglycerol kinase alpha monoclonal antibody DaMab-8 for immunohistochemical analyses. Monoclon Antib Immunodiagn Immunother 2020;39:1-5.
21. Nakano T, Ogasawara S, Tanaka T, Hozumi Y, Yamaki A, Sakane F, Shirai Y, Nakamura T, Yanaka M, Yamada S, Kaneko MK, Kato Y, and Goto K: DgMab-6: Antihuman DGKgamma monoclonal antibody for immunocytochemistry. Monoclon Antib Immunodiagn Immunother 2018;37: 229-232.

22. Nakano T, Ogasawara S, Tanaka T, Hozumi Y, Sano M, Sayama Y, Yamada S, Kaneko MK, Kato Y, and Goto K: DzMab-1: Anti-human diacylglycerol kinasezeta monoclonal antibody for immunocytochemistry. Monoclon Antib Immunodiagn Immunother 2019;38:179-182.

23. Sano M, Kaneko MK, and Kato Y: Epitope mapping of antidiacylglycerol kinase alpha monoclonal antibody DaMab-2. Monoclon Antib Immunodiagn Immunother 2019;38:8-11.

24. Sano M, Kaneko MK, and Kato Y: Epitope mapping of antihuman diacylglycerol kinase gamma monoclonal antibody DgMab-6. Monoclon Antib Immunodiagn Immunother 2019;38:120-123.

25. Sano M, Kaneko MK, and Kato Y: Epitope mapping of anti-diacylglycerol kinase zeta monoclonal antibody DzMab-1 for immunohistochemical analyses. Monoclon Antib Immunodiagn Immunother 2019;38:175-178.

26. Usuki T, Sakai H, Shionoya T, Sato N, and Sakane F: Expression and localization of type II diacylglycerol kinase isozymes delta and eta in the developing mouse brain. J Histochem Cytochem 2015;63:57-68.

27. Baum AE, Akula N, Cabanero M, Cardona I, Corona W, Klemens B, Schulze TG, Cichon S, Rietschel M, Nothen MM, Georgi A, Schumacher J, Schwarz M, Abou Jamra R, Hofels S, Propping P, Satagopan J, Detera-Wadleigh SD, Hardy J, and McMahon FJ: A genome-wide association study implicates diacylglycerol kinase eta (DGKH) and several other genes in the etiology of bipolar disorder. Mol Psychiatry 2008;13:197-207.

28. Kittel-Schneider S, Lorenz C, Auer J, Weissflog L, and Reif A: DGKH genetic risk variant influences gene expression in bipolar affective disorder. J Affect Disord 2016;198:148-157.

29. Isozaki T, Komenoi S, Lu Q, Usuki T, Tomokata S, Matsutomo D, Sakai H, Bando K, Kiyonari H, and Sakane F: Deficiency of diacylglycerol kinase eta induces lithium-sensitive mania-like behavior. J Neurochem 2016;138:448-456.

30. Nakano $T$, Iravani $A$, Kim $M$, Hozumi $Y$, Lohse $M$, Reichert E, Crotty TM, Stafforini DM, and Topham MK: Diacylglycerol kinase eta modulates oncogenic properties of lung cancer cells. Clin Transl Oncol 2014;16:29-35.

Address correspondence to: Yukinari Kato

New Industry Creation Hatchery Center Tohoku University

2-1, Seiryo-machi, Aoba-ku

Sendai

Miyagi 980-8575

Japan

E-mail: yukinari-k@bea.hi-ho.ne.jp

Received: March 15, 2020 Accepted: May 8, 2020 